INPLASY

PROTOCOL

To cite: Zhang et al. Can high frequency ultrasound combined computed tomography accurately diagnose thyroid tumor?: a protocol of systematic review and meta-analysis. Inplasy protocol 202040022. doi: 10.37766/inplasy2020.4.0022

Received: 04 April 2020

Published: 04 April 2020

Corresponding author: Lin-hua Zhang

Lin-huaZhang@outlook.com

Author Affiliation:

Ningbo Municipal Hospital of TCM

Support:

XYSTBSRP(2014K04-05)

Review Stage at time of this submission: The review has not yet started.

Conflicts of interest: None

\section{Can high frequency ultrasound combined computed tomography accurately diagnose thyroid tumor?: a protocol of systematic review and meta-analysis}

Zhang $\mathrm{LH}^{1}$; Chen G²; Tao W³.

Review question / Objective: Can high frequency ultrasound combined with computed tomography (HFUCT) accurately diagnose thyroid tumor (TT)?

Condition being studied: High frequency ultrasound; computed tomography; thyroid tumor.

Information sources: Electronic searches We will include any types of studies on assessing the clinical value of HFUCT for diagnosis of TT from any time period published to the present from the following databases: Cochrane Library, EMBASE, PubMed, Scopus, Web of Science, OpenGrey, Cumulative Index to Nursing and Allied Health Literature, Allied and Complementary Medicine Database, and China National Knowledge Infrastructure. No limitations of language and publication status will be imposed. We will also check reference lists of other relevant reviews, clinical trial registry, and conference proceedings.

INPLASY registration number: This protocol was registered with the International Platform of Registered Systematic Review and Meta-Analysis Protocols (INPLASY) on 04 April 2020 and was last updated on 04 April 2020 (registration number INPLASY202040022). 
Comparator: Reference test: We will include patients with histological-proven TT in the control group.

Study designs to be included: We will only consider case-controlled studies (CCS) on assess the clinical value of HFUCT for the diagnosis of TT. However, we will exclude any other.

Eligibility criteria: This study will include CCS that compared the clinical value of HFUCT for the diagnosis of TT with histological-proven TT.

Information sources: Electronic searches We will include any types of studies on assessing the clinical value of HFUCT for diagnosis of TT from any time period published to the present from the following databases: Cochrane Library, EMBASE, PubMed, Scopus, Web of Science, OpenGrey, Cumulative Index to Nursing and Allied Health Literature, Allied and Complementary Medicine Database, and China National Knowledge Infrastructure. No limitations of language and publication status will be imposed. We will also check reference lists of other relevant reviews, clinical trial registry, and conference proceedings.

Main outcome(s): The primary outcomes are sensitivity and specificity. The secondary outcomes are positive likelihood ratio, negative likelihood ratio, and diagnostic odds ratio.

Data management: Two authors will independently extract information for each study selected for final inclusion based on the prior designed sheet for data extraction. If there are any discrepancies between two authors, a third experienced author will be consulted to solve the different opinions. We will extract the following data: title, first author, publication time, location, patient characteristics, inclusion and exclusion criteria, study method, index test, refer test, outcomes, and funding information. If any insufficient information occurs, we will contact corresponding authors to inquire those data.
Quality assessment / Risk of bias analysis: We will use Quality Assessment of Diagnostic Accuracy Studies tool to assess study quality for each included study. This tool is utilized for evaluating diagnostic accuracy studies. Two authors will independently assess the study quality, and any divergences between two authors will be consulted a third author to make final decision.

Strategy of data synthesis: We will use RevMan V.5.3 software to synthesize the data and to perform data analysis. We will calculate outcome data using descriptive statistics and $95 \%$ confidence intervals. $I^{2}$ statistic will be used to check the degree of statistical heterogeneity among included studies with the guide judgments: $I^{2} \leq \mathbf{5 0 \%}$ indicating low heterogeneity, while $\mathrm{I}^{2}>\mathbf{5 0 \%}$ exerting obvious heterogeneity. We will use pool the data using a fixed-effect model, and we will consider performing metaanalysis to calculate pooled effect for outcome measurements if those eligible studies are sufficiently homogeneous $\left(\mathbf{I}^{2} \leq\right.$ $50 \%$ ). Otherwise, a random-effect model will be used and subgroup analysis will be carried out to identify the reasons those may cause obvious heterogeneity. In addition, we will plan to undertake a narrative description to synthesize data by reporting detailed written commentary to demonstrate the findings, target participant characteristics, and types of outcome measurements.

Subgroup analysis: We will operate subgroup analysis based on different study or patient characteristics, comparators, and outcomes.

Sensibility analysis: We will plan to perform sensitivity analysis by removing low quality studies to check the robustness of outcome results.

\section{Countries involved: China}

Keywords: High frequency ultrasound; computed tomography; thyroid tumor; sensitivity; specificity 